\title{
Non-Equilibrium Generation of Catalytic Supramolecular Polymers of Pre-RNA Nucleobases
}

\author{
Syed Pavel Afrose ${ }^{1}$, Chiranjit Mahato ${ }^{1}$, Pooja Sharma ${ }^{1}$, Lisa Roy ${ }^{2}$ and Dibyendu Das ${ }^{1 *}$
}

Bioenergetics played critical roles for the chemical emergence of life where available energy resources drove the generation of primitive polymers and fueled early metabolism. Further, apart from information storage, the catalytic roles of primitive nucleic acid fragments have also been argued to be important for biopolymer evolution. Herein, we have demonstrated the non-equilibrium generation of catalytic supramolecular polymers of a possible proto-RNA building block (melamine) driven by a thermodynamically activated ester of low molecular weight. We utilized reversible covalent linkage to install a catalytic imidazole moiety in the polymer backbone. This resulted in energy dissipation via hydrolysis of the substrate predominantly from the assembled state and subsequent disassembly, thus installing kinetic asymmetry in the energy consumption cycle. Noncatalytic analogues led to kinetically stable polymers while inactivated substrates were unable to drive the polymerization. The non-equilibrium polymers of the pre-RNA bases were capable to spatiotemporally bind to a model cofactor. Notably, presence of an exogenous aromatic base augmented the stability of the polymers, reminiscent to what the molecular midwives did during early evolution.

\footnotetext{
${ }^{1}$ Department of Chemical Sciences and Centre for Advanced Functional Materials, Indian Institute of Science Education and Research (IISER) Kolkata, Mohanpur 741246, India. Email: dasd@iiserkol.ac.in

${ }^{2}$ Institute of Chemical Technology Mumbai - IOC Odisha Campus Bhubaneswar, IIT Kharagpur Extension Centre, Bhubaneswar 751013, India.
} 
The entropically disfavoured state of living matter is characterized by the presence of genetic and catalytic polymers that exist in a non-equilibrium steady state sustained via continuous consumption of energy ${ }^{1-5}$ The importance of existing high energy nucleic acid polymers (NAPs) is ubiquitously recognized in numerous biochemical functions where duplication and information transfer are the central roles. Further, the RNA world hypothesis credits the intrinsic catalytic proficiency of existing NAPs to support their advent as the earliest proto-biopolymers. ${ }^{6}$ Yet the mechanism by which the kinetically stable but thermodynamically activated phosphodiester linkages have evolved is still unresolved. Just as extant biology uses thermodynamically activated phosphodiester monomers as the source of chemical energy for biopolymer synthesis in water, simple activated esters might have driven the assembly of non-equilibrium proto-RNAs and contributed to open ended evolution as suggested from the concept of dynamic kinetic stability. ${ }^{7}$ As a result of the greater availability of precursor organic bases in prebiotic soup, the proto-RNA eventually got substituted via iterations of chemical transformations under the energy driven non-equilibrium conditions. ${ }^{1,3,8,9}$

To support a general theory of biopolymer evolution that predated the emergence of life working far from equilibrium, a systems chemistry approach is essential to study a relatively simple set of monomers under a flux of energy and subsequently probe the possibility of generation of complex oligomers with useful and emergent properties such as catalysis. ${ }^{10-25} \mathrm{Far}$ from equilibrium behaviour involving repeated breaking and making of such simple precursors can result in temporal generation of aggregated structures with emergent functions which in principle could have assisted in selection and adaptation. ${ }^{3,8}$ Herein, we show the dissipative self-assembly of supramolecular polymers of protoRNA building blocks based on melamine that is driven by a thermodynamically activated ester. The imidazole functionalized melamine via a dynamic bond demonstrated acceleration of hydrolytic ability and subsequent dissipation from assembled state, thus installing kinetic asymmetry in the energy consumption pathways. ${ }^{8,26-29}$

As thermodynamically uphill polymerization in biology is accessed by activated ester, we started our investigation with the 4-nitrophenol (PNP) ester (MB*) of an organic acid (4-hydroxy-3methoxybenzoic acid, $\mathbf{M B}$ ) as the activated monomer. Melamine (MM) was used as the building block as it has been argued as the precursor of modern nucleobases (Fig. 1). ${ }^{9}$ The constituents were separately sparingly soluble in the aqueous medium having $83 \%(\mathrm{v} / \mathrm{v}$ ) buffer in DMSO (Supplementary information). However, when mixed together at 1:1 ratio with concentrations of $17 \mathrm{mM}$ each under the same conditions, we observed solubilization with gradual increase of viscosity. Eventually, a selfsupporting stable gel was observed (Supplementary Fig. 1). Transmission electron microscopy (TEM) used to characterize the self-assembled state of $\mathbf{M M - M B *}$ demonstrated long branched and linear fibrillar structures with very high aspect ratios (Supplementary Fig. 2). Notably, the thermodynamically stable precursor acid $\mathbf{M B}$ was unable to drive $\mathbf{M M}$ assembly till concentrations of $20 \mathrm{mM}$ and yielded a free-flowing solution in the same solvent system (Supplementary Fig. 3, TEM micrograph shown in Supplementary Fig. 4).

Contemporary catalytic RNA performs critical steps such as esterase activity, which is attributed to the nucleobases that help in the cleavage or ligation of the phosphodiester backbone. ${ }^{30} \mathrm{We}$ argued if the basicity of the system could be augmented, the dissipation of chemical energy via hydrolytic degradation of the ester and subsequent autonomous disassembly of the supramolecular polymers could be achieved. This could lead to the realization of dissipative non-equilibrium polymers of the protonucleobases. Utilizing a dynamic covalent bond, we reversibly mutated the melamine component of the polymer using 4-imidazolecarboxaldehyde (ImCHO, Fig. 1). Apart from featuring in the structure of modern purine nucleobases, imidazole mutated hairpin ribozymes have been shown to play important role in the cleavage of phosphodiesters. ${ }^{31}$ When equimolar (17 mM) concentrations of $\mathbf{M M}, \mathrm{MB}^{*}$ and ImCHO were taken as ternary mixture, the solution rapidly became viscous and turned into a gel within a minute (Fig. 2a). Imine formation was suggested from FTIR (Supplementary Fig. 5). The gel gradually began to turn yellow which suggested ongoing hydrolysis upon mixing. The 
yellow colour intensified with time and after $\approx 2.5 \mathrm{~h}$ the gel turned into a sol (Fig. $2 \mathrm{a}$ ). The morphology of the non-equilibrium assemblies was probed with time resolved electron microscopic techniques.

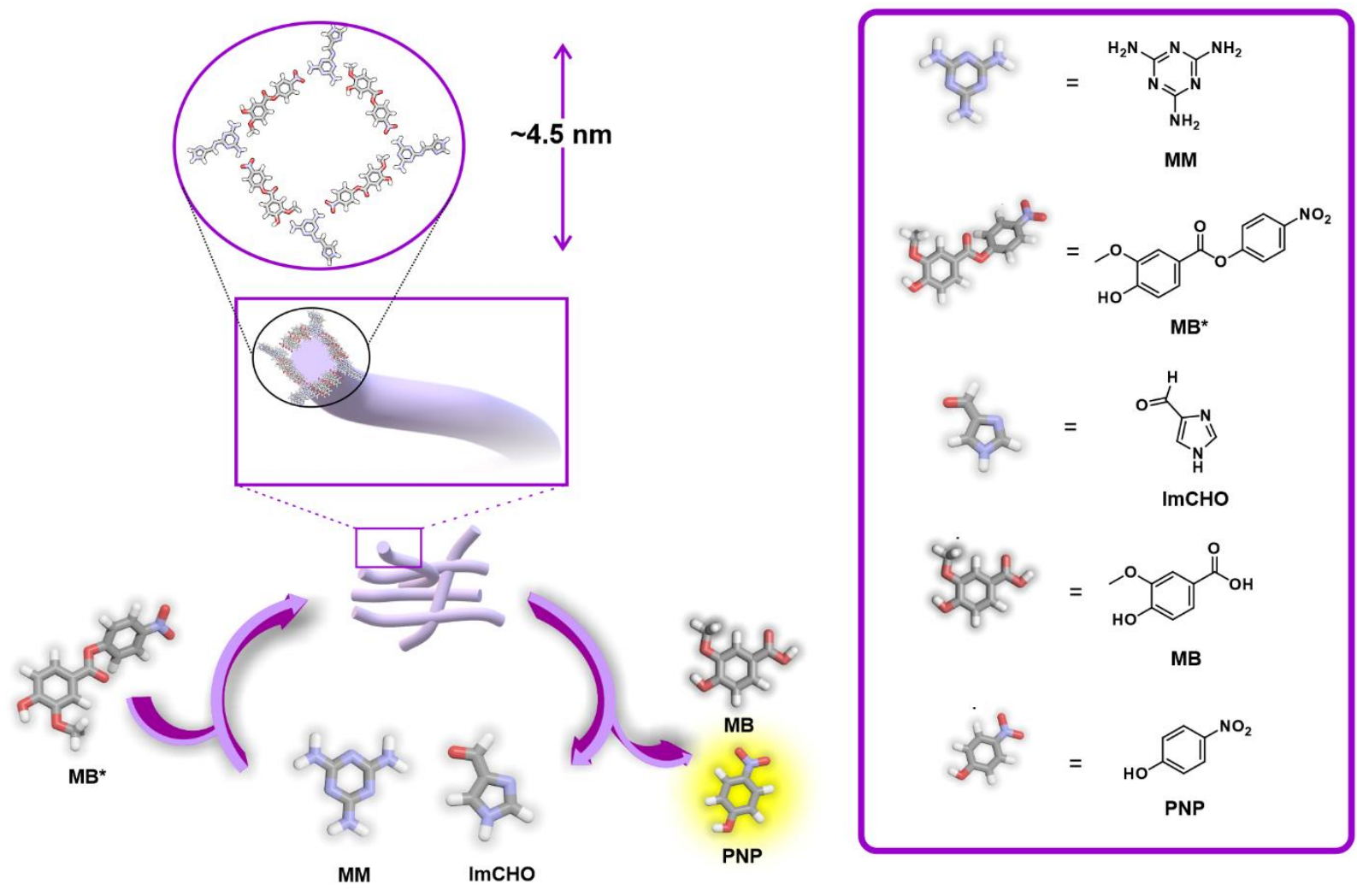

Fig. 1. Out of equilibrium supramolecular polymers of melamine (MM) driven by 4-nitrophenyl ester of 4-hydroxy-3-methoxybenzoic acid (MB*). Hydrolysis is accelerated from assembled state due to formation of a dynamic covalent linkage between $\mathbf{M M}$ and 4-imidazolecarboxaldehyde (ImCHO). Structures of the molecules are shown in the box. The polymerized state suggests a rosette like arrangement of the building blocks.

Transmission electron microscopy (TEM) done with the non-equilibrium system comprising of ImCHO with $\mathbf{M M}$ and driven by $\mathrm{MB}^{*}$ showed gradual formation of fibrillar structures. At $\mathrm{t}=15 \mathrm{~min}$, the intensification of the network like structures were observed suggesting the ongoing polymerization (Fig. 2c). TEM images of the ca. $2.5 \mathrm{~h}$ sample showed dissolution of the proto-biopolymer networks, consistent with the gel to sol transformation (Fig. 2d). Scanning electron microscopy (SEM) suggested the similar observations as the $\mathrm{t} \approx 0 \mathrm{~min}$ and $\mathrm{t}=2.5 \mathrm{~h}$ samples showed very little to no networks, while the $t=15$ min sample showed dense networks of fibres (Fig. 2e, Supplementary Fig. 6). Interestingly, from achiral building blocks, the generation of supramolecular helicity as twisted morphologies could be observed to be intensified in the non-equilibrium state from both TEM and SEM (Fig. $2 c$ inset and Fig. 2e). 


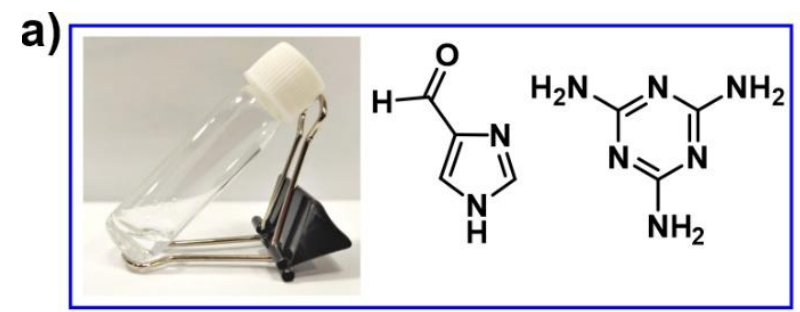<smiles>COc1cc(C(=O)Oc2ccc([N+](=O)[O-])cc2)ccc1O</smiles>
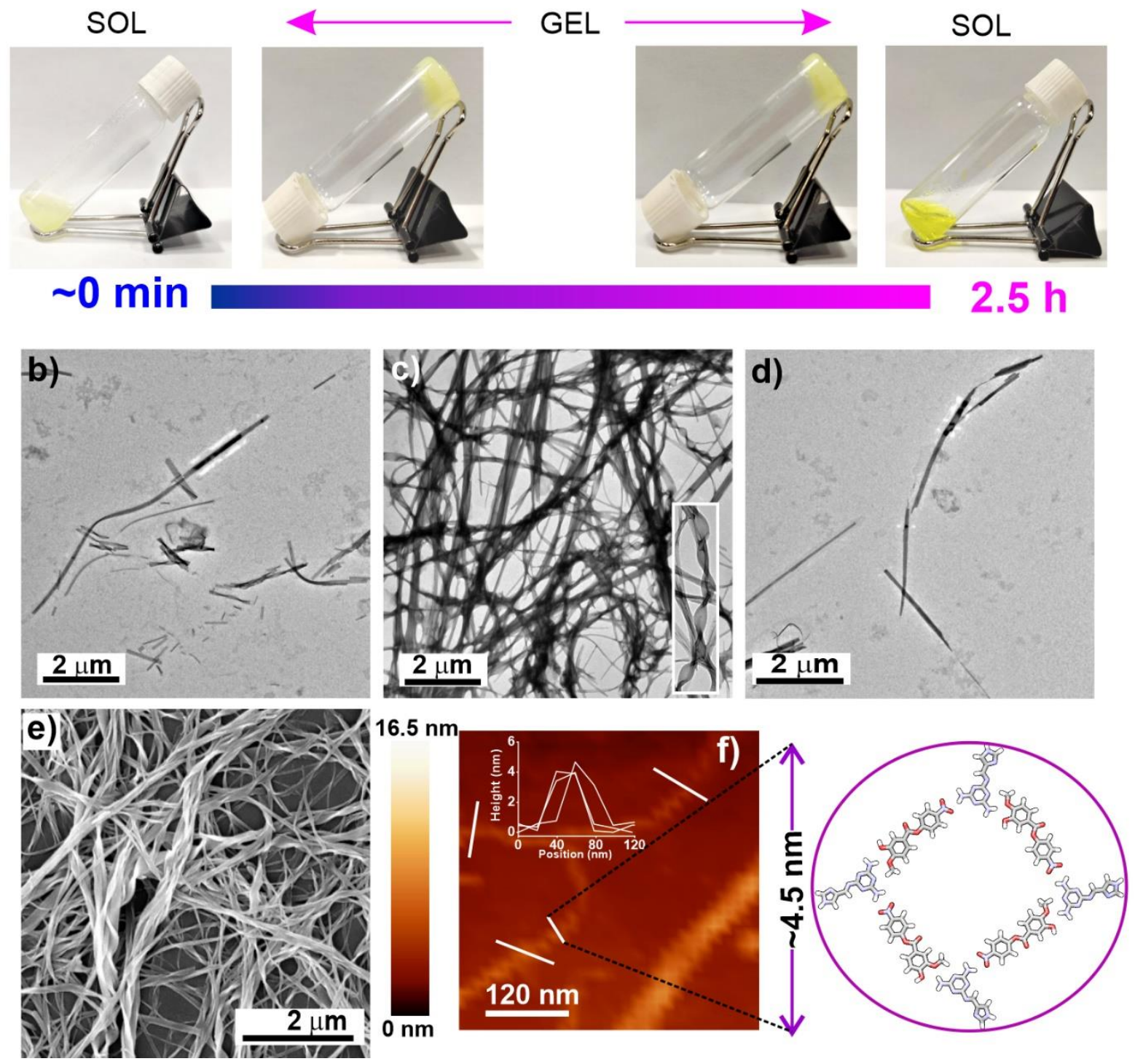

Fig. 2. a) Non-equilibrium phase transition and accessing of gel driven by $\mathbf{M B}^{*}$ in presence of ImCHO. Representative vial images at different time intervals, from $1 \mathrm{~min}$ to $2.5 \mathrm{~h}$ of addition of $\mathbf{M B}$ * Representative TEM micrographs of the non-equilibrium sample at $\mathrm{t} \approx 0 \mathrm{~h} \mathrm{(b),} 15 \mathrm{~min}$ (c) and $2.5 \mathrm{~h} \mathrm{(d)}$. Zoomed version of the helical morphology is shown in the inset of (c). e) Representative SEM micrograph of the non-equilibrium biopolymers at $t=15 \mathrm{~min}$. f) AFM topographic image of the nonequilibrium proto-biopolymers and the corresponding height profile (inset). Height of the fibres suggest a rosette like arrangement. 
Atomic force microscopy (AFM) height profile of the non-equilibrium proto-biopolymers showed a minimum height of $\approx 4.0-4.5 \mathrm{~nm}$ for fibres perpendicular to the image plane (Fig. 2f). This suggested cyclic rosette formation featuring four molecules of $\mathbf{M M}$ imine and four molecules of $\mathbf{M B}^{*}$ in an alternating manner (Fig. 2f). DFT studies further supported similar arrangement of these molecules with extended $\mathrm{H}$-bonding and $\pi-\pi$ interactions, leading to aggregates of rosette structures (Fig. 1 and Fig. $2 \mathrm{f}$ and Supplementary Fig. 7 for details). The fibres spanning a length of several micrometres suggested $\pi$ - $\pi$ stacking of such rosettes. Structures with larger diameter could be attributed to the aggregation of single fibres. Such aggregated structures were also observed from the TEM images where laterally aggregated bundles of the individual fibres were seen (Fig. 2c).

A good insight into the autonomous assembly and disassembly leading to sol-gel-sol transformations could be obtained from time dependent rheological measurements which can investigate temporal changes of the storage modulus. Frequency sweep measurements showed almost 200 percent increase in storage modulus in case of the non-equilibrium gel having $\mathbf{M M}, \mathbf{M B}^{*}$ and $\mathbf{I m C H O}$ compared to the one lacking ImCHO (only $\mathbf{M M}$ and $\mathbf{M B}^{*}$, Supplementary Fig. 8, 9). This suggested the participation of ImCHO in the polymer formation when premixed with $\mathbf{M M}$ and $\mathbf{M B}^{*}$. The temporal change of the mechanical strength could be observed from the time dependent rheology as the storage modulus showed an initial increase followed by a gradual decline, ultimately reaching a value close to the initial one ( $G^{\prime}$, Fig. 3b). The possible role of imidazole in catalyzing the hydrolysis step and concomitant energy dissipation was probed by using an abasic heterocycle (pyrrole-2-carboxaldehyde, PyrCHO, Fig. 3a) as a control instead of ImCHO. This control system accessed the self-supporting gel with similar network like morphology (Supplementary Fig. 10,11). Further, FTIR suggested imine formation between MM and PyrCHO (Supplementary Fig. 12). However, gel to solution transformation was not observed for prolonged time which suggested the importance of degradation of the high energy ester $\mathbf{M B}^{*}$ for dissipative self-assembly (Fig. 3a, Supplementary Fig. 10). The gel having the non-hydrolysing PyrCHO showed a similar increase of storage modulus over time due to ongoing polymerization (Fig. 3 b). Due to lack of catalytic potential, the increase in $\mathrm{G}^{\prime}$ was not accompanied by a subsequent decrease resulting from disassembly. The dissipative polymerization of the protonucleobases was further probed with a fluorophore 8-Anilinonaphthalene-1-sulfonic acid (ANS) which is known to report the microenvironment of assemblies upon binding. The emission of ANS (20 $\mu \mathrm{M}$ ) at $\approx 488 \mathrm{~nm}$ showed an increase of intensity up to $2 \mathrm{~h}$ (Fig. 3c, Supplementary Fig. 13). Afterwards, the intensity started to gradually decline till $6 \mathrm{~h}$. This value at ca. $6 \mathrm{~h}$ was even lower than the initial fluorescence intensity at ca. 2 min which suggested that the driven polymerization was rapid. Expectedly, the samples with the PyrCHO showed increase and plateauing of the intensity with time implying the kinetic stability of the assemblies (Fig. 3c).

To follow the catalytic activity of the non-equilibrium polymers, the hydrolytic degradation of the labile MB* ester was monitored by UV-Vis spectroscopy. With time, the absorbance at $405 \mathrm{~nm}$ showed an increase with a rate of $7.04 \pm 0.67 \mu \mathrm{M} . \mathrm{min}^{-1}$ (Fig. 3d). Control samples featuring PyrCHO showed miniscule background hydrolytic rate that was similar to what was observed for the stable gels of $\mathbf{M M}$ MB* in the absence of ImCHO (Fig. 3d). One of the important prerequisites of non-equilibrium dissipative self-assembly is the installation of kinetic asymmetry in energy consumption pathways that necessitates dissipation of energy predominantly from the high-energy assembled state. For this purpose, we first investigated the rates of hydrolysis of $\mathrm{MB}^{*}$ in presence of ImCHO only. Expectedly, due to the presence of imidazole moiety, we observed a modest hydrolytic rate of $0.59 \pm$ $0.3 \mu \mathrm{M} \cdot \mathrm{min}^{-1}$. However, this rate was more than an order of magnitude lower than what was observed for the dissipative gel (Fig. 3d). ${ }^{32-34}$ This implied that the formation of the polymers featuring the imidazole moieties resulted in acceleration of the rate of hydrolysis upon assembly and suggested energy dissipation from the self-assembled state. Further, we used unsubstituted imidazole which is a substantially stronger base but cannot form the dynamic linkage like ImCHO. Free imidazole indeed 
could rapidly hydrolyze $\mathrm{MB}^{*}$ in absence of $\mathrm{MM}$ with rates of $222.04 \pm 7.1 \mu \mathrm{M} . \mathrm{min}^{-1}$ which was 376 fold higher than the rate shown by free ImCHO. However, when we compared the rates in assembled state (with $\left.\mathrm{MM}-\mathrm{MB}^{*}\right)$, the rate of hydrolysis by imidazole $\left(\mathrm{V}_{\mathrm{i}}=5.9 \pm 0.75 \mu \mathrm{M} \cdot \mathrm{min}^{-1}\right)$ was lower than ImCHO (Table 1, Supplementary Information). This small but significant change of rates further underpinned the enhanced catalytic role of the ImCHO in degrading the ester from the assembled state which facilitated energy dissipation (Table 1, Supplementary Information). Despite being thermodynamically unstable, the resistance towards degradation in presence of strong heterocyclic bases underpins the unusual stability of the non-equilibrium polymers that could help them survive harsh environmental conditions; driving the system towards dynamic kinetic stability. ${ }^{7}$

An obstacle for the emergence of larger functional polymeric strands from short nucleotide fragments has been the inherent flexibility of the sequences which impede the polymerization process. Addition of planar intercalating molecules such as coralyne has been argued to facilitate strand polymerization by acting as midwives and hence providing a critical advantage for the polymer lifetime ${ }^{35} \mathrm{At}$ this point, we were curious whether the proto-biopolymers with long dimensions can recruit planar heterocyclic guests to prolong its lifetime. As a congener of coralyne, we chose isoquinoline to check its ability to prolong the lifetime of the non-equilibrium polymers. Interestingly, when $17 \mathrm{mM}$ of isoquinoline was premixed with $\mathbf{M M}, \mathbf{I m C H O}$ and $\mathbf{M B}^{*}$, the gels became stiffer and remained stable for more than two days, indicating the significant improvement of stability of the polymers (Fig. 4a). However, the rate of hydrolysis was not drastically affected by the inclusion of isoquinoline $\left(V_{i}=8.11 \pm 1.2 \mu \mathrm{M} . \mathrm{min}^{-1}\right.$, Supplementary Fig. 14). The stabilizing effect of isoquinoline was also reflected from rheological studies. Systems containing isoquinoline revealed greater mechanical strength with ca. 560 Pa higher $\mathrm{G}^{\prime}$ values compared to the non-equilibrium gel containing no isoquinoline (Supplementary Fig. 15, 16). Moreover, the high $G^{\prime}$ value was retained for a period of $24 \mathrm{~h}$ (Fig. 4b). NMR spectroscopy demonstrated that the aromatic proton signals of isoquinoline were not only broadened in the gel state but also showed a small upfield shift of $0.02 \mathrm{ppm}$ compared to the monomeric isoquinoline (Supplementary Fig. 17). This suggested possible inclusion of the heterocyle during the formation of the polymers.

The capability of primitive non-equilibrium RNA fragments to bind cofactors could be crucial for latent functions such as controlling primitive oscillatory rhythms and energy harvesting. We wondered whether the polymeric fibres with high aspect ratios can recruit small molecular cofactors and can temporally modulate their photophysical properties. We chose the hydrophobic planar dye Nile Red as a model cofactor with the expectation that the guest will be spatiotemporally entrapped. Time resolved confocal microscopy demonstrated non-equilibrium entrapment of the model cofactor within the assemblies. The micrographs of the $t=15 \mathrm{~min}$ samples incubated with Nile Red showed networks of fibres of high aspect ratio (Fig. $4 \mathrm{~d}$ ). The $\mathrm{t}=0 \mathrm{~min}$ and $\mathrm{t}=2.5 \mathrm{~h}$ samples did not show any noticeable formation of fibrous networks (Fig. 4c, 4e). Further proof of the entrapment was obtained from fluorescence lifetime imaging microscopy (FLIM) where the temporal generation of microenvironment on the proto-biopolymer was monitored. The binding event of the cofactor to the polymers was expected to induce a change in the average lifetime of the dye. At $t \approx 0 \mathrm{~min}$, the samples showed an average lifetime of $0.5 \mathrm{~ns}$ with little to no structures seen from the FLIM images. The $t=15$ min samples revealed the presence of fibres having moderate non-homogenous distribution of lifetimes close to $1.7 \mathrm{~ns}$ (Fig. 4f, inset). Finally, samples aged for ca. $2.5 \mathrm{~h}$ showed a histogram of an average lifetime of around $0.9 \mathrm{~ns}$, suggesting the release of the entrapped cofactor into the solution (Fig. 4f, inset). In order to demonstrate the temporal binding and release of the model cofactor, samples incubated with $10 \mu \mathrm{M}$ cofactor and aged for different times were mixed with an insoluble organic phase (ethyl acetate, Fig. 4g, Supplementary Information for details). UV-Vis spectroscopy of the organic phase revealed rapid initial release of the cofactor. However, around ca. $30 \mathrm{~min}$, the sample showed a decline in dye release suggesting stronger binding of the cofactor within the supramolecular polymers (Fig. 4g). However, the samples aged for ca. $1 \mathrm{~h}$ showed pronounced recovery of the absorbance intensities indicating release of the cofactor due to autonomous disassembly. Controls 
done with the kinetically stable gel of PyrCHO expectedly showed a sustained release of the entrapped dye.

a)<smiles>Nc1nc(N)nc(N)n1</smiles>

b)

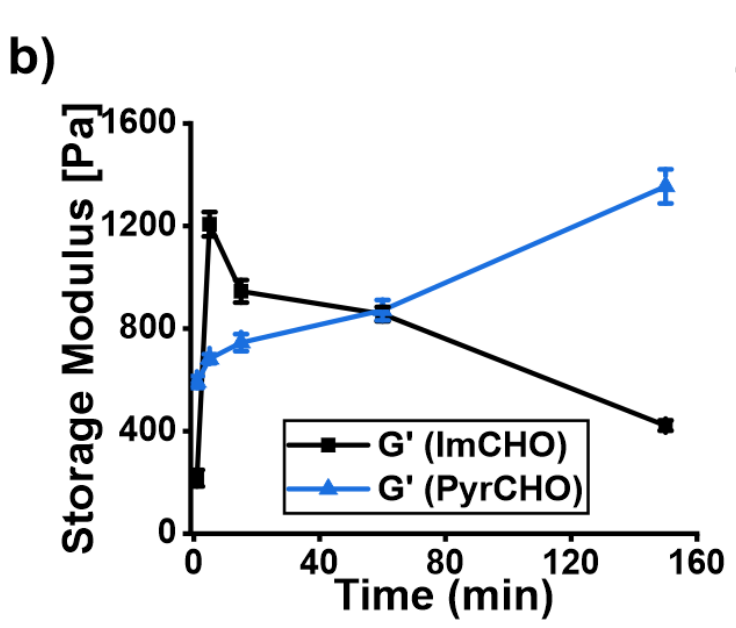

d)

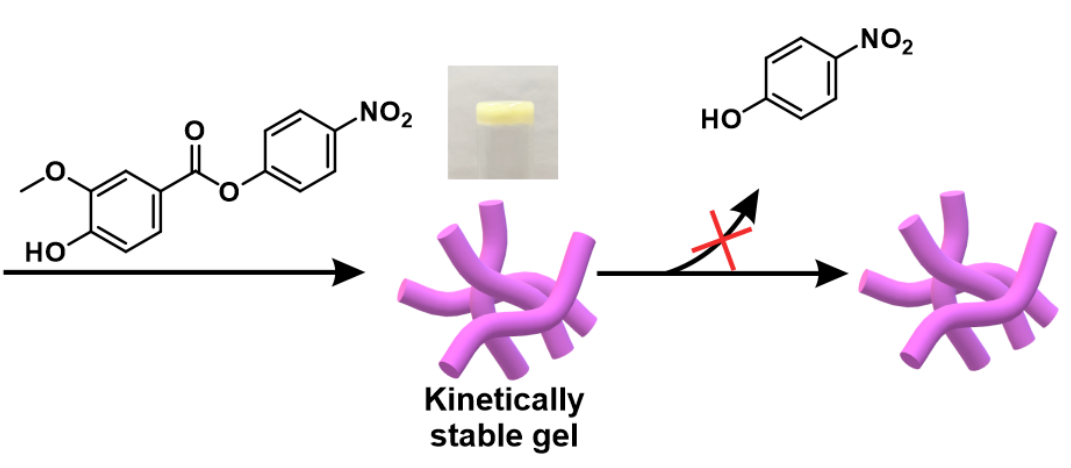

c)
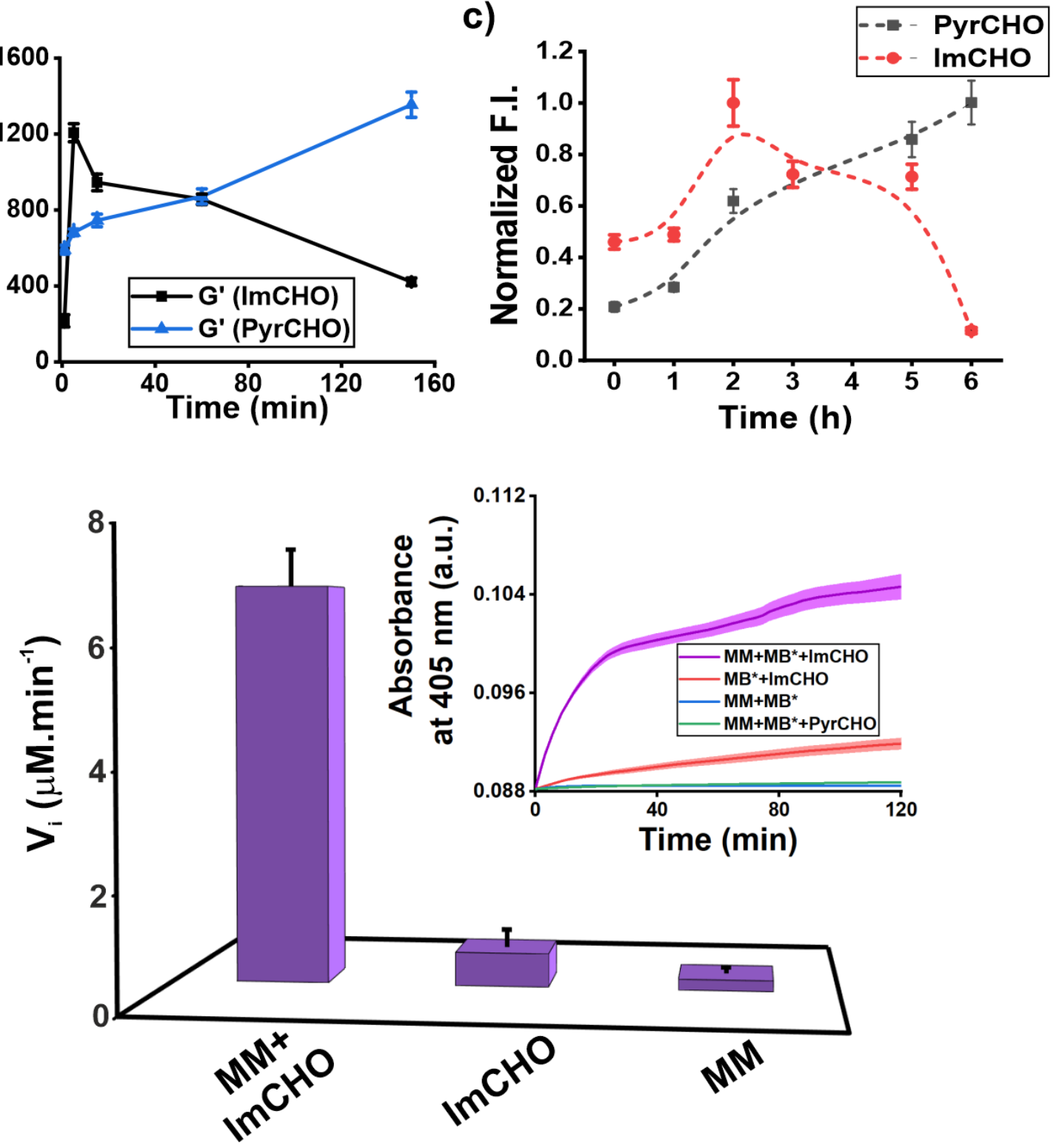

Fig. 3. a) Scheme showing formation of kinetically stable polymers in presence of PyrCHO. The incapability of PyrCHO to hydrolyze MB* leads to stability of the gel. b) Variation of storage modulus of different systems with time. c) Time dependent normalized fluorescence intensities at $488 \mathrm{~nm}$ of the ImCHO containing gel and the control PyrCHO gel. d) Bar diagram showing the initial velocities of 
hydrolysis of $\mathbf{M B}^{*}$ in different component mixtures. Inset shows time course kinetics of hydrolysis of $\mathbf{M B}^{*}$ in presence of MM and ImCHO (purple curve), only ImCHO (red curve), MM and PyrCHO (green curve) and only MM (blue curve). Error bars and error bands represent the standard deviations of three different measurements.

a)

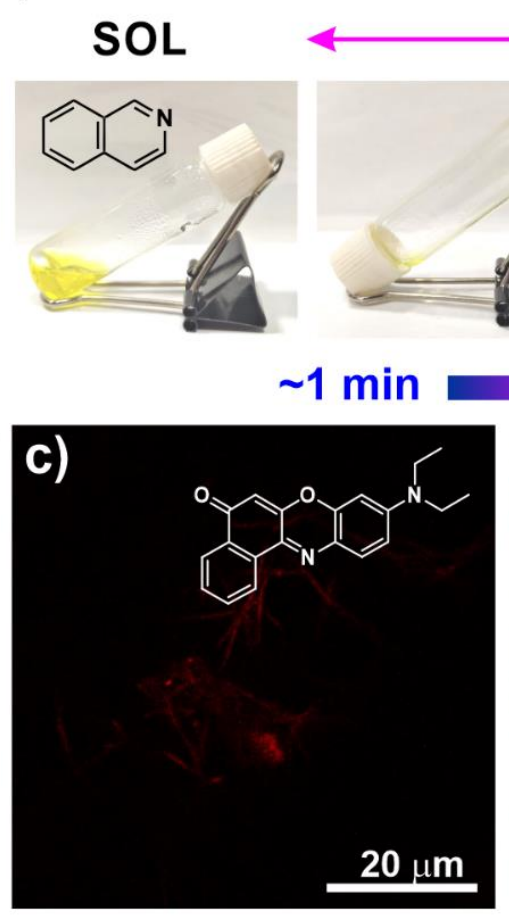

f)

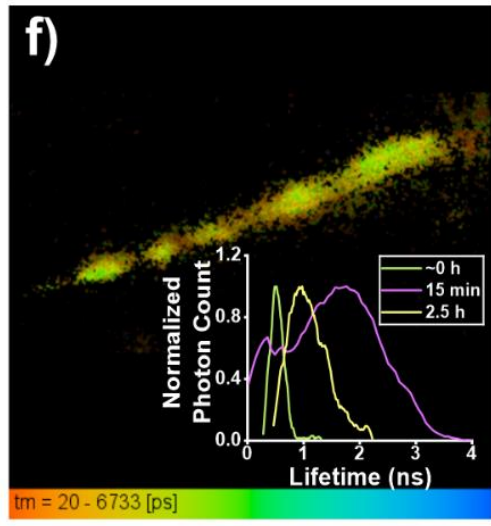

b)
GEL

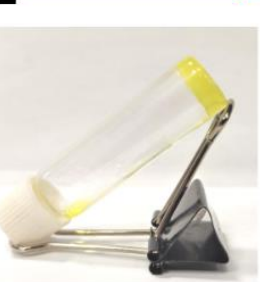

$24 \mathrm{~h}$
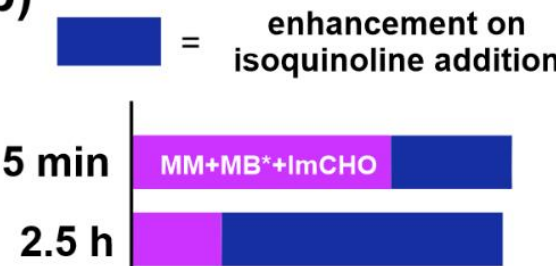

$24 \mathrm{~h}$

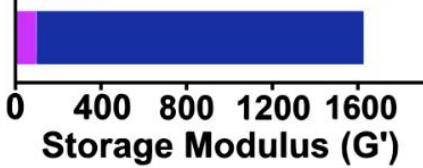

d)

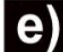

e)

$20 \mu \mathrm{m}$

$20 \mu \mathrm{m}$

g)
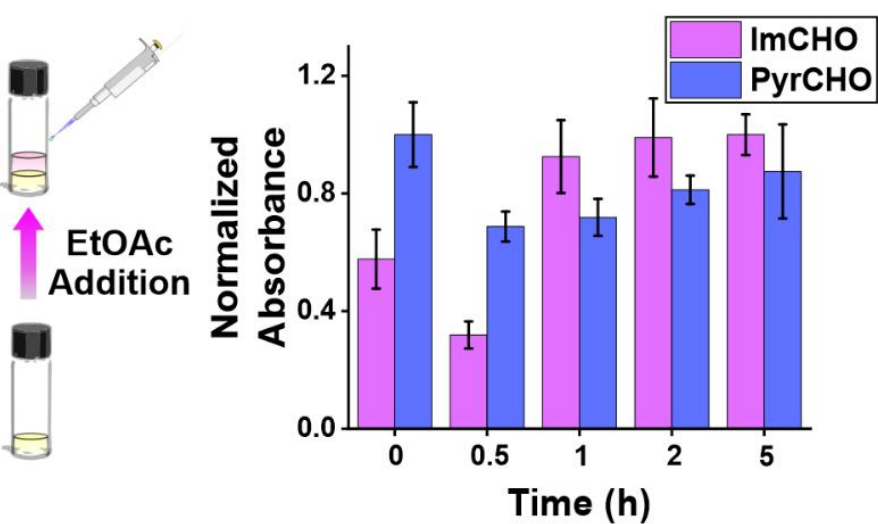

Fig. 4. a) Enhancement of lifetime of the non-equilibrium gel in presence of isoquinoline (inset: structure of isoquinoline). b) Bar diagram showing the storage modulus at time intervals of $5 \mathrm{~min}, 2.5$ $\mathrm{h}$ and $24 \mathrm{~h}$ of the sample containing MM, MB* and ImCHO (pink band) and the corresponding enhancements after inclusion of isoquinoline (blue band). Representative confocal micrographs of the non-equilibrium sample incubated with Nile red at $\mathrm{t} \approx 0 \mathrm{~h}$ (c), $15 \mathrm{~min}$ (d) and $2.5 \mathrm{~h}$ (e). f) Representative FLIM image of the non-equilibrium sample at $t=15 \mathrm{~min}$, incubated with Nile red. Distributions of average fluorescence lifetimes for samples at $\mathrm{t}=0 \mathrm{~h}, 15 \mathrm{~min}$ and $2.5 \mathrm{~h}$ are shown in the histogram (inset). g) Release of Nile red to the water insoluble organic phase (ethyl acetate) added on top of the assembly. Bar diagram showing the normalized absorbance intensities of Nile red at $526 \mathrm{~nm}$ in the organic phase for the ImCHO and PyrCHO containing systems. Error bars and error bands represent the standard deviations of three different measurements. 
Life being far from equilibrium, involves continuous consumption of energy to run the advanced biological supramolecular apparatuses. ${ }^{36,37}$ Chemical evolution that predated the emergence of life, required energy sources to access primitive ribosomal machineries which worked away from equilibrium. Herein, we have demonstrated the non-equilibrium generation of protonucleobases that are driven by activated ester-based monomers and involve non-covalent interactions responsive to environmental perturbations. These pre-RNA polymers were capable of undergoing reversible condensation with small heterocyclic bases and displayed emergent catalytic competence, subsequently leading to degradation of the substrate that drove the assembly. The catalytic ability showed significant enhancement upon driven polymerization and thereby featured a kinetic asymmetry in the energy consumption pathway. Controls without the covalent modification as well as with an abasic heterocycle showed significantly lower substrate consumption rates. Additionally, the non-equilibrium polymers of the pre-RNA bases were capable to spatiotemporally recruit guests for different benefits and functions. For instance, apart from the catalytic roles, the protonucleobases were capable to temporally bind a model cofactor which could in principle facilitate latent functions such as primitive oscillatory rhythms and energy harvesting. Further, the polymers could recruit planar aromatic heterocycles to remarkably improve the lifetime of its non-equilibrium state without significantly influencing the rate of dissipation.

Despite being more ephemeral than the kinetically locked networks of extant covalent nucleic acids, these non-equilibrium polymers were sufficiently robust for catalytic competence, recruiting beneficial molecular guests and in principle are positioned to support an emerging metabolism capable of chemical evolution and pushing the systems towards dynamic kinetic stability. ${ }^{7,38}$ Far from equilibrium polymers that involve making and breaking of high energy bonds and dynamic covalent linkages have the potential for stochastic innovation and can in principle undergo the process of selection and adaption. Considering the long dimensions of proto-biopolymers, it should be possible to integrate additional catalytic sites and molecular recognition elements within the polymer backbone which can set the stage for complex functions required for early metabolism and open-end evolution. ${ }^{39}$

\section{References}

1. Deamer, D. \& Weber, A. L. Bioenergetics and life's origins. Cold Spring Harb. Perspect. Biol. 2, a004929 (2010).

2. Whitesides, G. M. \& Grzybowski, B. Self-assembly at all scales. Science 295, 2418-2421 (2002).

3. England, J. L. Dissipative adaptation in driven self-assembly. Nat. Nanotechnol. 10, 919-923 (2015).

4. Astumian, R. D. Stochastic pumping of non-equilibrium steady-states: how molecules adapt to a fluctuating environment. Chem. Commun. 54, 427-444 (2018).

5. Hess, H. \& Ross, J. L. Non-equilibrium assembly of microtubules: from molecules to autonomous chemical robots. Chem. Soc. Rev. 46, 5570-5587 (2017).

6. Scott, W. G., Szöke, A., Blaustein, J., O'Rourke, S. M. \& Robertson, M. P. RNA catalysis, thermodynamics and the origin of life. Life 4, 131-141 (2014).

7. Pascal, R., Pross, A. \& Sutherland, J. D. Towards an evolutionary theory of the origin of life based on kinetics and thermodynamics. Open Biol. 3, 130156 (2013).

8. Ragazzon, G. \& Prins, L. J. Energy consumption in chemical fuel-driven self-assembly. Nat. Nanotechnol. 13, 882-889 (2018).

9. Cafferty, B. J. et al. Efficient self-assembly in water of long noncovalent polymers by nucleobases analogs. J. Am. Chem. Soc. 135, 2447-2450 (2013).

10. Ashkenasy, G., Hermans, T. M., Otto, S. \& Taylor, A. F. Systems chemistry. Chem. Soc. Rev. 46, 2543-2554 (2017).

11. Azevedo, H.S., Perry, S.L., Korevaar, P.A. et al. Complexity emerges from chemistry. Nat. Chem. 12, 793-794 (2020). 
12. Merindol, R. \& Walther, A. Materials learning from life: concepts for active, adaptive and autonomous molecular systems. Chem. Soc. Rev. 46, 5588-5619 (2017).

13. De, S. \& Klajn, R. Dissipative self-assembly driven by the consumption of chemical fuels. Adv. Mater. 282, 1706750 (2018).

14. Semenov, S., Wong, A., van der Made, R. et al. Rational design of functional and tunable oscillating enzymatic networks. Nat. Chem. 7, 160-165 (2015).

15. Debnath, S., Roy, S., \& Ulijn, R. V. Peptide nanofibers with dynamic instability through nonequilibrium biocatalytic assembly. J. Am. Chem. Soc. 135, 16789-16792 (2013).

16. Maity, I., Wagner, N., Mukherjee, R. et al. A chemically fueled non-enzymatic bistable network. Nat. Commun. 10, 4636 (2019).

17. Bal, S., Das, K., Ahmed, S. \& Das, D. Chemically fueled dissipative self-assembly that exploits cooperative catalysis. Angew. Chem. Int. Ed. 58, 244-247 (2019).

18. Muñana, P. S. et al. Substrate-induced self-assembly of cooperative catalysts. Angew. Chem. Int. Ed. 57, 16469-16474 (2018).

19. Afrose, S. P., Bal, S., Chatterjee, A., Das, K. \& Das, D. Designed negative feedback from transiently formed catalytic nanostructures. Angew. Chem. Int. Ed. 58, 15783-15787 (2019).

20. van der Helm, M. P. et al. Organocatalytic Control over a Fuel-driven Transient Esterification Network. Angew. Chem. Int. Ed. 59, 20604-20611 (2020).

21. Gentile, K., Somasundar, A., Bhide, A. \& Sen, A. Chemically Powered Synthetic "Living" Systems. Chem 6, 2174-2185 (2020).

22. Boekhoven, J., Hendriksen, W. E., Koper, G. J., Eelkema, R. \& van Esch, J. H. Transient assembly of active materials fueled by a chemical reaction. Science 349, 1075-9 (2015).

23. Tian, L. et al. Non-equilibrium spatiotemporal sensing within acoustically patterned twodimensional protocell arrays. ACS Cent. Sci. 4, 1551-1558 (2018).

24. Bal, S., Ghosh, C., Ghosh, T., Vijayaraghavan, R. K. \& Das, D. Non-equilibrium polymerization of cross- $\beta$ amyloid peptides for temporal control of electronic properties. Angew. Chem. Int. Ed. 59, 13506-13510 (2020).

25. Fanlo-Virgós, H., Alba, A. R., Hamieh, S., Colomb-Delsuc, M. \& Otto, S. Transient substrateinduced catalyst formation in a dynamic molecular network. Angew. Chem. Int. Ed. 53, 1134611350 (2014).

26. Astumian, R. D. Kinetic asymmetry allows macromolecular catalysts to drive an information ratchet. Nat. Commun. 10, 3837 (2019).

27. Penocchio, E., Rao, R. \& Esposito, M. Thermodynamic efficiency in dissipative chemistry. Nat. Commun. 10, 3865 (2019).

28. Chen, R., Neri, S. \& Prins, L. J. Enhanced catalytic activity under non-equilibrium conditions. Nat. Nanotechnol. 15, 868-874 (2020).

29. Caplow, M. \& Shanks, J. Mechanism of the microtubule GTPase reaction. J. Biol. Chem. 265, 8935-8941 (1990).

30. Piccirilli, J. A., McConnell, T. S., Zaug, A. J., Noller, H. F. \& Cech, T. R. Aminoacyl esterase activity of the Tetrahymena ribozyme. Science 256, 1420-1424 (1992).

31. Vázquez-Salazar, A., Becerra, A. \& Lazcano, A. Evolutionary convergence in the biosyntheses of the imidazole moieties of histidine and purines. PLoS One 13, e0196349 (2018).

32. Omosun, T., Hsieh, MC., Childers, W. et al. Catalytic Diversity in Self-propagating peptide assemblies. Nat. Chem. 9, 805-809 (2017).

33. Ottelé, J., Hussain, A. S., Mayer, C. \& Otto, S. Chance emergence of catalytic activity and promiscuity in a self-replicator. Nat. Catal. 3, 547-553 (2020).

34. Singh, N., Conte, M. P., Ulijn, R. V., Miravet, J. F. \& Escuder, B. Insight into the esterase like activity demonstrated by an imidazole appended self-assembling hydrogelator. Chem. Commun. 51, 13213-13216 (2015). 
35. Horowitz, E. D. et al. Intercalation as a means to suppress cyclization and promote polymerization of base-pairing oligonucleotides in a prebiotic world. Proc. Natl. Acad. Sci. US A. 107, 5288-5293 (2010).

36. Epstein, I. R. Reaction: life is messy. Chem 5, 1922-1923 (2019).

37. Bai, Y. et al. Achieving biopolymer synergy in systems chemistry. Chem. Soc. Rev. 47, 5444-5456 (2018).

38. Pross, A. How can a chemical system act purposefully? Bridging between life and non-life. J. Phys. Org. Chem. 21, 724-730 (2008).

39. Xavier, J. C., Hordijk, W., Kauffman, S., Steel, M. \& Martin, W. F. Autocatalytic chemical networks at the origin of metabolism. Proc. R. Soc. B 287, 20192377 (2020).

\section{Acknowledgment}

D.D. is thankful to SJF Grant SB/SJF/2020-21/08, GOI for financial assistance. SPA acknowledges UGC for fellowship and CM acknowledges Nano Mission (SR/NM/NS-1082/2015). We thank Arunavo Chatterjee for his valuable help.

\section{Author Contribution}

D.D. conceived and supervised the overall project. S.P.A and C.M. conceived and performed all the experiments. P.S. performed the AFM experiments. L.R. conceived and carried out the DFT studies. D.D., S.P.A and C.M. co-wrote the paper. All authors discussed the results and commented on the manuscript.

Corresponding Author

dasd@iiserkol.ac.in

ORCID Dibyendu Das:0000-0001-6597-8454

Table of content: 


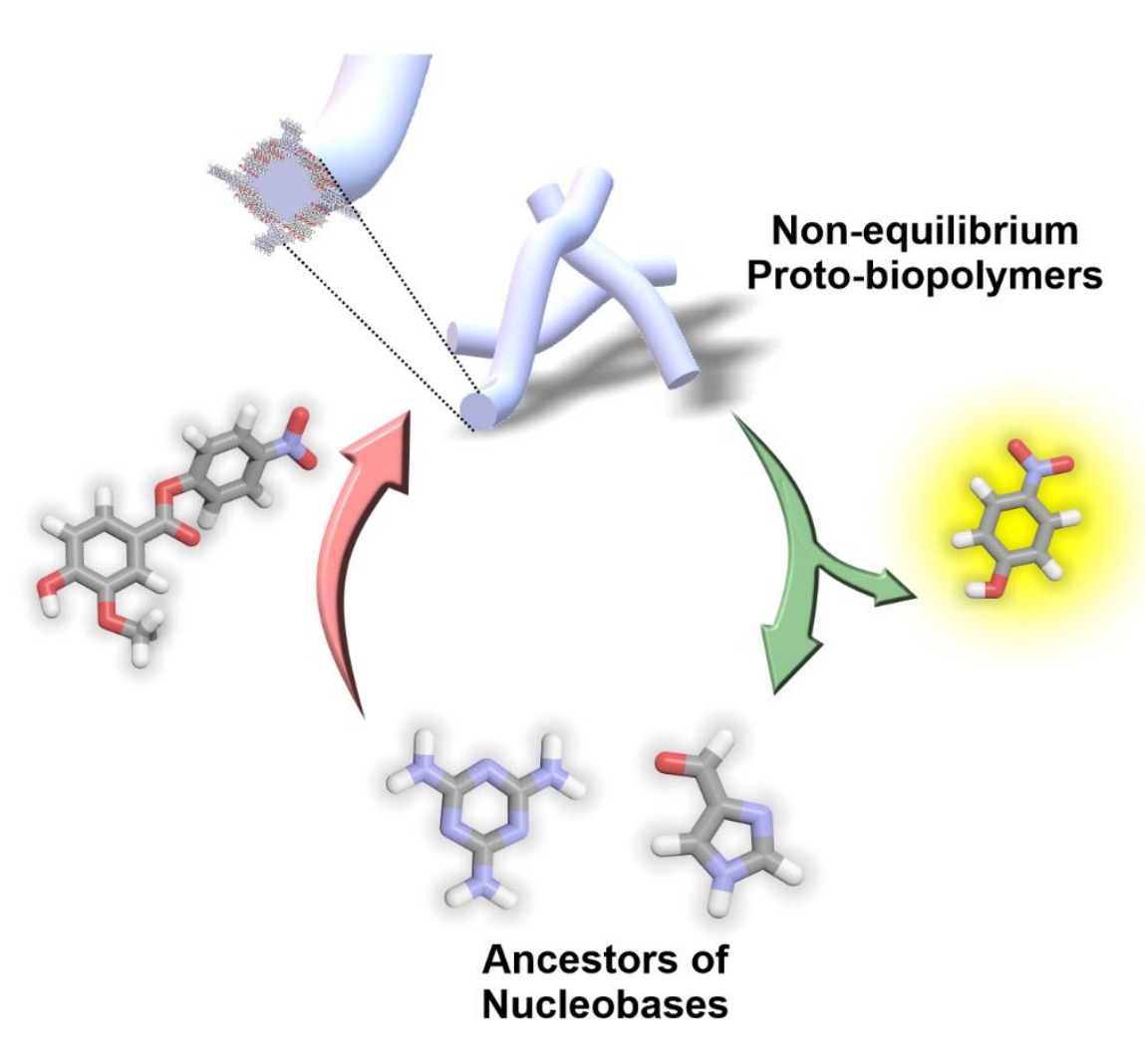

